Research Article

\title{
Prediction of the VIV Responses Based on the Numerical Solutions of Controlled Motion
}

\author{
Duanmu Yu $(\mathbb{D}$, Chen Jianping, and Chen Yizhong \\ Department of Ship and Port Eng., Guangzhou Maritime University, Guangzhou, Guangdong, China \\ Correspondence should be addressed to Duanmu Yu; duanmuyu1023@163.com
}

Received 27 April 2021; Revised 15 July 2021; Accepted 12 October 2021; Published 11 November 2021

Academic Editor: Yu xiang Ma

Copyright ( $\odot 2021$ Duanmu Yu et al. This is an open access article distributed under the Creative Commons Attribution License, which permits unrestricted use, distribution, and reproduction in any medium, provided the original work is properly cited.

\begin{abstract}
The study of forced and free vibration of a cylinder has long been isolated. The internal relationship between free vibration and forced vibration has rarely been investigated. In this paper, the relationship between the forced and free vibration of a cylinder was established. A series of numerical simulations of a cylinder undergoing forced oscillations at a wide range of vibration amplitudes and frequencies were carried out, with the flow solver viv-FOAM-SJTU developed based on the open-source platform OpenFOAM. Complex demodulation analysis was conducted to quantify the spatial-temporal phase relationship between the forces and the displacement of the cylinder. It was found that, at some particular oscillating amplitudes and frequencies, the phase angle switched between positive and negative values, which corresponds to a vortex mode transferring from the $2 P$ mode to the $2 P_{O}$ mode. This distinct new mode " $2 P_{O}$ " was closely related to the intermittent jumping between lower and upper branches of the amplitude responses of VIV. A prediction model was developed to obtain the VIV amplitude responses based on the numerical results of forced oscillation. The prediction results of three points located separately in the initial, upper, and lower branches of VIV agreed well with experimental measurements of an elastically mounted cylinder. This prediction model was thus expected to be suitable for predicting the response of VIV.
\end{abstract}

\section{Introduction}

When a flow passes a cylinder, the oscillatory shedding of vortices into the wake would cause fluctuating lift and drag forces on the cylinder. If the cylinder was elastically mounted, the fluctuating forces could excite the vibrations of the cylinder. The motions of the cylinder would in turn change the vortex wake. Such a problem of fluid-structure interaction was called vortex-induced vibrations (VIV). VIV was a highly specialized subject that incorporates fluid mechanics, structural mechanics, vibrations, computational fluid dynamics (CFD), etc. and occurs in many engineering situations such as marine riser pipes, mooring cables, tethered structures, and spar hulls. VIV could cause large stresses and fatigue damage of structures. So far, numerous contributions to flow-induced oscillations in general and to VIVs in particular had guided the acquisition of design data through physical and numerical experiments, theoretical, and physical research work, of which many had been discussed in the compressive reviews of Williamson [1]; Williamson [2]; Williamson [3]; Sarpkaya [4]; Gabbai [5]; Bearman [6]; Wu [7]. Much progress had been made during the past decade, both numerically and experimentally, toward the understanding of the kinematics of VIV. Studies for VIV could be divided into two categories: free vibration of a cylinder and forced vibration of a cylinder.

For free vibrations of a cylinder, the vibration force was provided through the fluid forces exerted on the cylinder. Mass ratio $m^{*}$ was an important parameter that influences the amplitude response of VIV. Feng's [8] experiment of free vibration of an elastically mounted cylinder in air, which represents the typical model of high mass ratio, was the earliest and perhaps the most famous experiment. According to Feng [8], there were two branches for the amplitude response: the vortex mode changes from $2 S$ of the initial branch to $2 P$ of the lower branch. The situation for low mass ratio was quite different. A new branch, called the upper branch, appeared in the amplitude response of VIV for low 
mass ratio [9-12], besides the initial and lower branches, as was also shown for the high mass ratio. The maximum response amplitude reached about one diameter (1D) of the cylinder, which was greater than Feng's results (0.6D). Jauvtis and Williamson [13] focused their attention on the study of vortex-induced vibration with two degrees of freedom. The amplitude response obtained from combined $\mathrm{X}$ and $\mathrm{Y}$ motion was larger than $\mathrm{Y}$-only motion ( $\mathrm{X}$ is the inline direction, and $\mathrm{Y}$ is the cross-flow direction). A new vortex wake mode " $2 \mathrm{~T}$," which consisted of a triple of vortices, was observed for low mass ratios VIV experiments $\left(m^{*}=2.6\right)$. Other researchers carried out numerical simulations of freely oscillating cylinder with CFD methods. Navrose and Sanjay [14] reported free vibrations of a cylinder of low mass ratio $m^{*}=10$ at Reynolds number $\mathrm{Re}=1000$. Three branches were obtained, and the maximum amplitude of cross-flow (y) oscillations was 0.7D. Al-Jamal and Dalton [15] performed a series of 2-D LES simulations of the VIV response of a circular cylinder at $\mathrm{Re}=8000$ with a wide range of damping ratios and natural frequencies. Lucor et al. [16] presented DNS results of uniform flow past a rigid cylinder with low mass damping at $\mathrm{Re}=1000,2000$, and 3000. Wanderley et al. [17] correctly predicted the amplitudes of the VIV oscillations by solving the slightly compressible Reynolds average NS equations with the RoeSweby scheme.

For forced vibrations, the cross-flow (y) movement of the cylinder was predefined by the sinusoidal trajectory relative to the fluid. Williamson [18] conducted experiments of controlled oscillation, over a broad range of amplitudes ( $A^{*}$ up to 5.0) and wavelengths $\left(\lambda^{*}\right.$ up to 15.0). The results showed principally three vortex wake patterns, named $2 S$, $2 P$, and $P+S$ modes, which were relevant to the fundamental synchronization regime. These experiments had been used as benchmarks and have been verified by experiments or numerical simulations conducted by later researchers. Williamson and Roshko [18] studied the cross-flow (y) forced vibration of a circular column in uniform flow. Nishihara et al. [19] presented the characteristics of fluid forces and wake patterns of a circular cylinder oscillating in the streamwise direction in a cross-flow. Jeon and Gharib [20] performed the experiments of a cylinder undergoing one-and two-degree-of-freedom motions and found that the addition of streamwise motion will result in qualitative changes in the wake. Numerical simulations were carried out as an alternative method to study forced oscillations of a circular cylinder. Atluri et al. [21] investigated the influence of Reynolds number (ranging from 500 to 8000) on the wake structure of a forced oscillating cylinder at different vibration frequencies. Dong and Karniadakis [22] presented DNS results of the flow past an oscillating cylinder at $\mathrm{Re}=10000$ by employing a multilevel-type parallel algorithm. Zhao and Wan $[23,24]$ presented DES results of the flow past an oscillating cylinder. Fu et al. [25] presented VIVs of a flexible cylinder in an oscillatory flow. Deng et al. [26] studied VIVs of a flexible cylinder by three-dimensional strip model.

As was shown in the foregoing studies, the study on the forced and free vibration had long been isolated. The internal relationship between free vibrations and forced vibrations had received few investigations. However, the ultimate objective of VIV research was to predict, to the extent possible, the kinematics and dynamics of self-excited vibrations from forced vibration (physical/numerical) experiments and, equally important, the dynamics of forced oscillation (say, e.g., drag lift, and inertia coefficients and the phase angle) from the physical/numerical experiments with self-excited oscillations [4]. Morse and Williamson [27] performed 5680 runs of forced oscillating experiments in a water flume and accurately predicted the response and wake modes for vortex-induced vibration response. A new distinct mode of vortex formation, termed as the $2 P_{\text {OVERLAP }}$ mode $\left(2 P_{O}\right)$, was identified. This mode also consisted of two pairs of vortices (like $2 P$ ), but one vortex in each pair was much weaker than the other. The extreme care of control required for forced vibration experiments and extensive measurements needed to create very high-resolution data make it arduous to accomplish such experimental studies [27]. Moreover, many factors such as running condition, installation error, and collection of data could influence the experimental results. Compared to experiments, numerical simulations can easily control the simulation parameters and obtain the detailed vortex structures. One objective of our study was to identify the new vortex mode $2 P_{O}$ by numerical simulations. The new vortex mode was closely related to intermittent jumping between lower and upper branches of the amplitude responses of VIV. We found that the wake could switch intermittently between the $2 P$ and $2 P_{O}$ modes, even if the cylinder was vibrating with constant amplitude and frequency. In other words, the two different vortex modes could exist simultaneously at some specific oscillating amplitudes and frequencies, contrary to the conventional notion that only one clear vertex formation can exist. Complex demodulation analysis was used to obtain the time history of the phase difference between the lift coefficient and the displacement. The phase angle switched between positive and negative values, corresponding to the change of vortex mode transition between $2 P$ mode and $2 P_{O}$ mode. Another objective of this study was to predict the amplitude response of free vibration cylinders from forced vibration. The prediction function was derived based on the concept of an "energy balance" to compute the amplitude response of a free oscillating cylinder at different mass damping ratios. Our work helped explain several important questions of free vibrations of a cylinder.

This paper presented a number of numerical researches of the forced oscillating cylinder at $\mathrm{Re}=4000$. The simulations were carried out with a newly developed flow solver viv-FOAM-SJTU, which was integrated with six-degree-offreedom motion module and coupled dynamic deformation mesh module, based on open-source platform OpenFOAM. All the simulations were performed by solving the Reynoldsaveraged Navier-Stokes (RANS) equations with the SST $k-$ $\omega$ turbulence model. The finite volume method (FVM) for spatial discretization and implicit Euler scheme for temporal discretization were adopted. The pressure-velocity coupling was dealt with by the PIMPLE (merged PISO-SIMPLE) algorithm, a large time-step transient solver for incompressible flow. 
This paper is organized as follows. Section 2 gives an introduction of numerical methods, which were composed by four parts. In section 2.1, the numerical fluid model is introduced firstly. All the nondimensionalized parameters used in the paper are presented in section 2.2. Next, the complex demodulation method used to obtain the phase angle between the lift force and displacement is introduced in section 2.3. The prediction model, which is used to obtain the VIV amplitude responses based on the numerical results of forced oscillation, is described in section 2.4. Section 3 presents and discusses the numerical results. First, an overview of the computational domain was presented, and four different mesh verifications were carried out to confirm the mesh convergence. Next, numerical simulations of forced oscillation with constant frequency $\lambda^{*}=6.2$ and different amplitude $A^{*}$ ranging from 0.4 to 0.9 were carried out. And discussions about the wake modes and vortex transition were carried out to verify the new $2 P_{O}$ mode, which would give the explanation for the intermittent switch between the lower branch and upper branch. The last part of section 3 was about the validation of the prediction model by using the free vibration responses of Govardhan and Williamson [28]. The VIV responses were successfully predicted based on the forced vibration data with three typical cases $\lambda^{*}=5.0,6.0,6.2$, which were located in the initial, upper, and lower branches separately. Finally, a summary of the paper is presented.

\section{Numerical Method}

2.1. Numerical Fluid Model. The fluid motion is represented by the incompressible unsteady Reynolds-Averaged Navier-Stokes (URANS) equations. Details of the solution procedure implemented in OpenFOAM are described in Jasak [29] and Rusche [30]; and only a brief introduction was presented here.

The Reynolds-averaged Navier-Stokes equations are as follows:

$$
\begin{gathered}
\frac{\partial \overline{u_{i}}}{\partial x_{i}}=0 \\
\frac{\partial}{\partial t}\left(\rho \overline{u_{i}}\right)+\frac{\partial}{\partial x_{j}}\left(\rho \overline{u_{i} u_{j}}\right)=-\frac{\partial \bar{p}}{\partial x_{i}}+\frac{\partial}{\partial x_{j}}\left(2 \mu \overline{S_{i j}}-\rho \overline{u_{j}^{\prime} u_{i}^{\prime}}\right),
\end{gathered}
$$

where $\overline{S_{i j}}=1 / 2\left(\partial \overline{u_{i}} / \partial x_{j}+\partial \overline{u_{j}} / \partial x_{i}\right)$ is the mean rate of strain tensor. $-\rho \overline{u_{j}^{\prime} u_{i}^{\prime}}$ results from the fluctuating velocity field and is generally referred to as the Reynolds stress $\tau_{i j}$.

A turbulent model is required to compute the Reynolds stresses for turbulence closure. The SST $k-\omega$ turbulence model is used because of its well performance in reverse pressure gradient and separated flow. The Reynolds stress is modelled by a linear constitutive relationship using Boussinesq hypothesis with the mean flow straining field as

$$
\tau_{i j}=-\rho \overline{u_{j}^{\prime} u_{i}^{\prime}}=2 \mu_{t} \overline{S_{i j}}-\frac{2}{3} \rho k \delta_{i j},
$$

where $\mu_{t}$ is the turbulent viscosity, obtained from solving the SST $k-\omega$ model, and $k=(1 / 2) \overline{u_{i}^{\prime} u_{i}^{\prime}}$ is the mean turbulent kinetic energy.
Applying equations (3) to (2), one has

$$
\frac{\partial}{\partial t}\left(\rho \overline{u_{i}}\right)+\frac{\partial}{\partial x_{j}}\left(\rho \overline{u_{i}} \overline{u_{j}}\right)=-\frac{\partial \overline{p_{\mathrm{eff}}}}{\partial x_{i}}+\frac{\partial}{\partial x_{j}}\left(2 \mu_{\mathrm{eff}} \overline{S_{i j}}\right),
$$

where $p_{\text {eff }}=p+2 / 3 \rho k, \mu_{\text {eff }}=\mu+\mu_{t}$

The solution of the governing equations is achieved by using the PIMPLE, which is a combined PISO-SIMPLE algorithm and is adapted for large time-step computation of incompressible flow.

2.2. Basic Parameters. All the parameters are nondimensionalized, so that different systems can be compared with each other. The nondimensional parameters of the paper are shown in Table 1.

2.3. Complex Demodulation. The phase angle $\varphi$ between the fluid lift force and cylinder displacement is determined by a complex demodulation of the lift coefficient and displacement. A description of the technique is given as follows:

The lift coefficient signal is chosen as the reference signal, as

$$
C_{L}(t)=C_{L 0} \cos \left(2 \pi f_{\mathrm{ex}} t+\varphi(t)\right),
$$

where $C_{L 0}$ is the amplitude of the lift coefficient, $\varphi$ is the angle between the cylinder displacement and fluid lift, and $f_{\text {ex }}$ is the frequency of forced oscillation.

$$
\begin{aligned}
C_{L}(t) & =C_{L 0} \cos \left(2 \pi f_{\mathrm{ex}} t+\varphi(t)\right) \\
& =\frac{1}{2} C_{L 0}\left[\exp \left\{i\left(\omega_{\mathrm{ex}} t+\varphi(t)\right)\right\}+\exp \left\{-i\left(\omega_{\mathrm{ex}} t+\varphi(t)\right)\right\}\right],
\end{aligned}
$$

where $\omega_{\mathrm{ex}}=2 \pi f_{\mathrm{ex}}$ is the angular frequency.

Demodulation by multiplying by $\exp \left(-i \omega_{\mathrm{ex}} t\right)$,

$$
\begin{aligned}
Y(t) & =C_{L}(t) \exp \left\{-i \omega_{\mathrm{ex}} t\right\} \\
& =\frac{1}{2} C_{L 0} \exp \{i \varphi(t)\}+\frac{1}{2} C_{L 0} \exp \left\{-i\left(2 \omega_{\mathrm{ex}} t+\varphi(t)\right)\right\} .
\end{aligned}
$$

$$
\cdot(a)(b)
$$

Low-pass filter to remove frequencies at or above frequency $\omega_{\text {ex }}$ : this removes term (b), and smooth term (a). The result is

$$
Y^{\prime}(t)=\frac{1}{2} C_{L 0}^{\prime} \exp \left\{i \varphi^{\prime}(t)\right\}
$$

Extract $\varphi^{\prime}(t): \exp \left\{i \varphi^{\prime}(t)\right\}=2 Y^{\prime} / C_{L 0}^{\prime}$.

2.4. Prediction Model. The equation, which represents the vortex-induced vibrations of a cylinder oscillating in the cross-flow (y) direction, is defined as follows:

$$
m \ddot{y}+c \dot{y}+k y=F_{L} .
$$

According to Table $1, F_{L}=1 / 2 C_{L} \rho U^{2} D L$, and then, the vibration equations can be expressed as 
TABLE 1: Definition of dimensionless variables.

\begin{tabular}{lcc}
\hline Reynolds number & $\operatorname{Re}$ & $U D / \nu$ \\
\hline Mass ratio & $m^{*}$ & $m /\left(\pi \rho(D / 2)^{2} L\right)$ \\
Damping ratio & $\zeta$ & $c / 2 \sqrt{\mathrm{mk}}$ \\
Amplitude ratio & $A^{*}$ & $A / D$ \\
Normalized wavelength & $\lambda^{*}$ & $\lambda / D=U /\left(f_{\mathrm{ex}} D\right)$ \\
Lift force coefficient & $C_{L}$ & $2 F_{L} /\left(\rho U^{2} D L\right)$ \\
Drag force coefficient & $C_{D}$ & $2 F_{D} /\left(\rho U^{2} D L\right)$ \\
Frequency ratio & $f^{*}$ & $f_{\mathrm{ex}} / f_{n}$ \\
\hline
\end{tabular}

In Table 1, $U$ is the inlet velocity; $f_{\text {ex }}$ is the forced oscillating frequency; $f_{n}$ is the natural frequency in water; $D$ is the cylinder diameter; $L$ is the cylinder length; $v$ is the fluid kinematic viscosity; $\rho$ is the fluid density; $m$ is the cylinder mass; $c$ is the structural damping; $k$ is the spring constant; $F_{L}$ is the lift force of the cylinder; $F_{D}$ is the drag force of the cylinder.

$$
m \ddot{y}(t)+c \dot{y}(t)+k y(t)=\frac{1}{2} C_{L}(t) \rho U^{2} D L .
$$

The parameters in the equation are shown in Table 1 . The cylinder motion $y(t)$, which is given by the controlled vibration, is a precisely sinusoidal function. The lift force coefficient $C_{L}(t)$, caused by uniform flow around a controlled vibrating cylinder, is also approximated by a sinusoidal function

$$
\begin{aligned}
y(t) & =A \sin \left(2 \pi f_{\mathrm{ex}} t\right), \\
C_{L}(t) & =C_{L 0} \sin \left(2 \pi f_{\mathrm{ex}} t+\varphi\right),
\end{aligned}
$$

where $A$ is the amplitude of the controlled motion, and $C_{L}(t)$ can be resolved into a component in phase with the cylinder velocity $C_{L v}$, and a component in phase with the cylinder acceleration $C_{L a}$

$$
C_{L}(t)=C_{L v} \cos \left(2 \pi f_{\mathrm{ex}} t\right)+C_{L a}\left(-\sin \left(2 \pi f_{\mathrm{ex}} t\right)\right),
$$

where $C_{L v}=C_{L 0} \sin \varphi, C_{L a}=-C_{L 0} \cos \varphi$.

The structural damping cand spring constant kare defined as $c=2 \zeta m \omega_{n} k=m \omega_{n}^{2}$, where $\omega_{n}=2 \pi f_{n}$, and $f_{n}$ is the natural frequency of the circular cylinder in water. Substituting $c, k, Y=y /$ Dinto equation (11) combined with nondimensional variables, then equation (11) can be expressed as

$$
\ddot{Y}+2 \zeta \dot{Y}+Y=\frac{C_{L} \rho U^{2}}{2 \omega_{n}^{2} m} .
$$

Transforming equations (13) and (14) to the dimensionless form and substituting them into

$$
\frac{A}{D}=\frac{1}{4} \frac{C_{L 0} \sin \varphi}{\pi^{3} m^{*} \zeta}\left(\frac{U}{f_{\mathrm{ex}} D}\right)^{2} \frac{f_{\mathrm{ex}}}{f_{n}} .
$$

The prediction equation is given as follows, by applying Table 1:

$$
C_{L 0} \sin \varphi=\frac{4 \pi^{3} A^{*} m^{*} \zeta}{\lambda^{* 2} f^{*}}
$$

Different from experimental studies, added-mass coefficient is not considered in numerical simulations. Instead, the contribution of pressure and viscous forces is solved by
$\mathrm{N}-\mathrm{S}$ equations directly. The influence of added mass is considered by the fluid force in the right-hand side of N-S equations.

The prediction method is based on the balance of the energy. The term $C_{L 0} \sin \varphi$ is the force coefficient in phase with the velocity. This term is denoted as the "fluid excitation" and represents a normalized energy into the system. For a free-vibration system oscillating at steady state this energy must be balanced by the normalized energy out of the system. The phase angle $\varphi$ is an important parameter that influences the transition of energy from the fluid flow the cylinder oscillation. If the phase angle $\varphi$ is between $0^{\circ}$ and $180^{\circ}$, then it will yield positive excitation from fluid to cylinder. If the phase angle $\varphi$ is between $-180^{\circ}$ and $0^{\circ}$, then it will yield negative excitation from cylinder to fluid.

\section{Computational Results and Discussion}

3.1. Grid Convergence Discussion. In this part, grid convergence is verified to check the mesh of forced oscillation simulations. We present a study of flow dynamics associated with simulated two-dimensional flows past a circular cylinder that is in simple harmonic cross-flow (y) oscillation. The cylinder oscillation in the cross-flow (y) direction is expressed by

$$
y=A \cos \left(2 \pi f_{\mathrm{ex}} t\right)
$$

Results are examined for $\operatorname{Re}=4000$ and a fixed motion amplitude of $A^{*}=A / D=0.4$, and a fixed vibration frequency of $\lambda^{*}=U_{0} / f_{\mathrm{ex}} D=4$.

Computational geometry is two-dimensional, as shown in Figure 1. The length of computational geometry is from $-10 D$ of the boundary inlet to $20 D$ of the boundary outlet. The width is from $-15 D$ of the boundary top to $15 D$ of the boundary bottom.

Four different mesh verifications were carried out by changing the nodes around the cylinder and the distance of nearest grid to the cylinder boundary. Table 2 summarizes the information of the four meshes. Figure 2 shows the whole gird and mesh information around the cylinder. All grids used in this paper are structured grids, which is easy for systematic refinement in all directions.

Table 3 summarizes the values of the time average of drag coefficient $\overline{C_{D}}$ and lift coefficient amplitude $\overline{C_{L \max }}$, the vortex shedding frequency $f_{s t}$, the Strouhal number $S t$, and the phase angle $\varphi$ at four grid resolutions from the simulation for the flow past an oscillating cylinder at $\mathrm{Re}=4000$. It can be seen that large differences in the grid size only slightly change the results. The Strouhal number is the same for all grids. It indicates that dominant frequency of vortex shedding shifts to the frequency of oscillating cylinder. At this condition, the lock-in of frequency will occur. The differences for the drag coefficient are smaller than $1.1 \%$, and the differences for the lift coefficient are smaller than $0.8 \%$. The phase angle is stable around $3.5^{\circ}$. Then, following this grid sensitivity study, it is dependable to proceed with the study of vortex mode transitions and prediction the free vibration responses by employing controlled oscillations. 


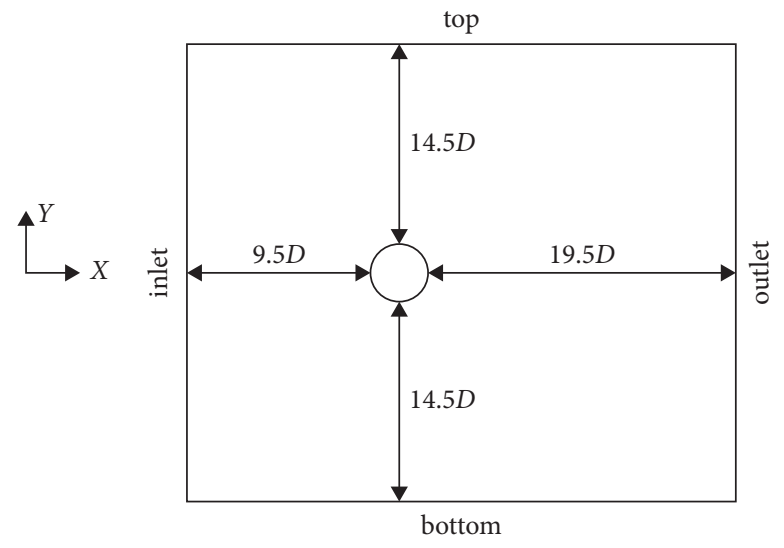

FIGURE 1: The calculational geometry.

TABLE 2: The details of the mesh for the computation.

\begin{tabular}{lccc}
\hline Mesh & Number of cells & Number of nodes around the cylinder & The height of nearest grid to the cylinder boundary \\
\hline Mesh I & 21900 & 120 & $0.004 D$ \\
Mesh II & 29300 & 120 & $0.003 D$ \\
Mesh III & 46500 & 160 & $0.003 D$ \\
Mesh IV & 71600 & 200 & $0.002 D$ \\
\hline
\end{tabular}

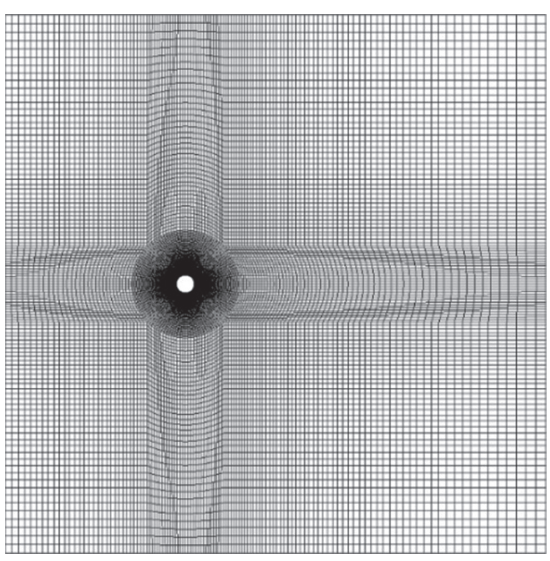

(a)

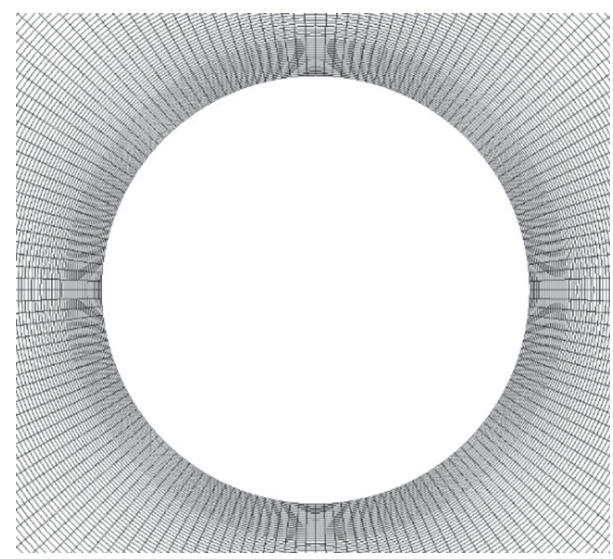

(b)

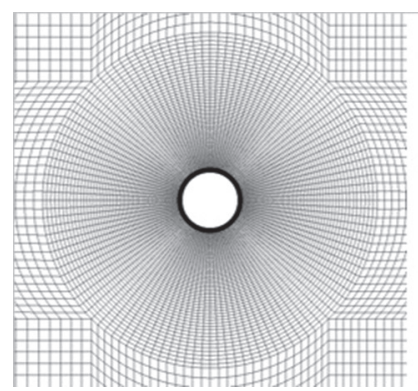

I

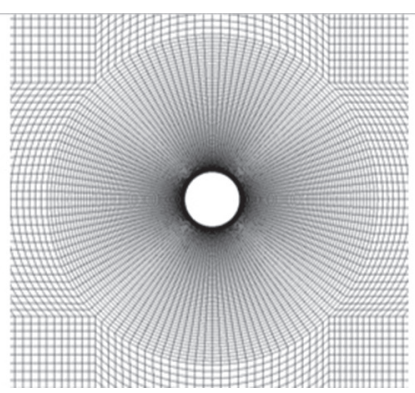

II

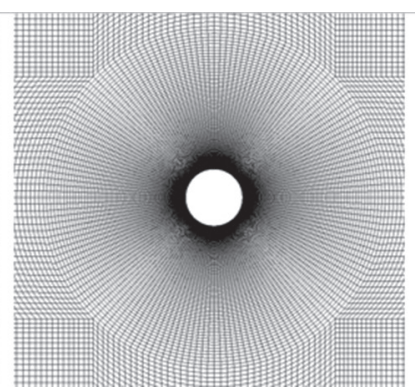

III

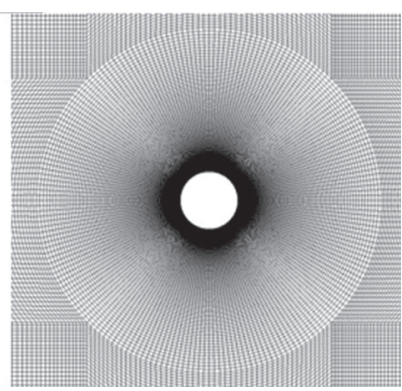

IV

(c)

FIGURE 2: (a) Uniform mesh for the global domain; (b) boundary layers around cylinder; (c) details of the four meshes around the cylinder. 
TABLe 3: Force coefficient and phase between lift and displacement.

\begin{tabular}{lccccc}
\hline Mesh & $\overline{C_{D}}$ & $\overline{C_{L \max }}$ & $f_{s t}$ & $S t$ & $\varphi\left[^{\circ}\right]$ \\
\hline Mesh I & 1.4306 & 2.4484 & 0.691 & 0.25 & -3.6786 \\
Mesh II & 1.4148 & 2.4592 & 0.691 & 0.25 & -3.4629 \\
Mesh III & 1.4145 & 2.4685 & 0.691 & 0.25 & -3.4916 \\
Mesh IV & 1.4144 & 2.4689 & 0.691 & 0.25 & -3.4957 \\
\hline
\end{tabular}

According the mesh convergence study, the mesh III is chosen for the next numerical simulations.

3.2. Wake Modes and Transitions. In this section, a forced motion cylinder controlled by sinusoidal oscillation with different amplitudes and a constant frequency is simulated to study the wake modes and transitions.

Williamson and Roshko [18] studied the vortex wake patterns of an oscillating cylinder controlled by a sine function. They researched different vortex modes and clarified them into three different principal types: $2 S$ pattern (each period alternatively shedding single vortex), $2 P$ pattern (each period alternatively shedding vortex pairs), and $P+S$ pattern (each period alternatively shedding single vortex and vortex pairs), which are shown in Figure 3(a). It identified the clear boundaries of the three principal vortex patterns.

Morse and Williamson [27] did 5680 controlled oscillation experiments over a more extensive range of normalized amplitude and wavelength. The map of vortex shedding regimes is shown in Figure 3(b). For the boundaries described by Williamson and Roshko [18], the transitions of the boundary separating different vortex patterns were clear and abrupt. However, Morse and Williamson [27] found several regions where two distinct vortex-formation modes can exist. In these regions, a distinct new mode of vortex formation was named " $2 P_{\text {OVERLAP," abbreviated as }}$ " $2 P_{O}$ " mode. The $2 P_{O}$ mode exists in its own clearly defined region of the amplitude-wavelength plane, overlapping the boundary between the $2 S$ and $2 P$ regions.

Many researchers have performed experiments or numerical simulations to verify the $2 S, 2 P$, and $P+S$ modes. However, no research work has been conducted to verify the new distinct mode pattern $2 P_{O}$. Therefore, it is essential for us to perform numerical simulations to verify the $2 P_{O}$ mode. A series of simulations about a forced oscillating cylinder with constant frequency $\lambda^{*}=6.2$ and different amplitude $A^{*}$ ranging from 0.4 to 0.9 are carried out.

First, phase angle $\varphi$ between the fluid lift force and cylinder displacement is studied by the method of complex demodulation to understand the system and its mechanism for vortex mode transitions. Figure 4 displays the time history of the $y$-direction motion, lift coefficient, and the phase angle at $\lambda^{*}=6.2, A^{*}=0.4$. As shown in Figure 4 , the phase angle $\varphi$ maintains a stable value of $140.0^{\circ}$. The vorticity contours of the obtained $2 P$ mode are shown in Figure 5. A pair of vortices, where the two vortices do not separate at all, shed alternatively from each side of the cylinder. Vorticity contours in this paper are defined by $Q$ criterion: $Q=1 / 2\left(|\Omega|^{2}-|S|^{2}\right)$. Here, $\Omega=1 / 2\left(\partial u_{i} / \partial x_{j}-\partial u_{j} / \partial x_{i}\right)$ is the vorticity; $S=1 / 2\left(\partial u_{i} / \partial x_{j}+\partial u_{j} / \partial x_{i}\right)$ is the strain invariant.
When amplitude $A^{*}$ of vibration is added from 0.5 to 0.9 , while oscillating frequency holds constant as before, we further study the vortex mode transitions with the changes of phase angle. Time histories of the phase angle are shown in Figure 6. This figure shows that, for amplitude $A^{*}=0.5$, the phase angle keeps at $159.7^{\circ}$, while, for the other four amplitudes increasing from 0.6 to 0.9 , the phase angle cannot remain unchanged as above. Abrupt jumps from a negative angle to a positive angle are observed. After a short time, the angle instantly goes back to its original negative value. The intermittent switch of angle appears to be irregular and unstable.

Accompanied with the jump of the phase angle, the vortex wake pattern is also changed. Take the dotted box in Figure 7 as an example. As the phase angle jumps to the positive part, the vortex wake exhibits a different pattern, as shown in Figure 6. This new pattern, named $2 P_{O}$, is first found by Morse and Williamson [27] by physical experiments. The new pattern is distinguished with the $2 P$ mode in that although two pairs of vortices alternatively shed each period, the strengths of the two vortices are unequal. One vortex in a pair is much weaker and decays quickly than the other. Figure 8 shows the $2 P_{O}$ mode vortex field obtained by digital particle image velocity (DPIV) measurements of Morse and Williamson [27]. The numerical simulations of the vortex patterns are consistent with the experimental results, as displayed in Figures 9 and 10. The good details of vortex street and vortex transition are presented. The very weak secondary vortex can only be observed in a very near region behind the cylinder. The weak vortex diffuses quickly when propagating downstream, which explains why many experimental researchers did not identify the $2 P_{O}$ mode before; one would confuse the $2 P_{O}$ mode with the $2 S$ mode, which is decided by how well one could solve the secondary weak vortex. However, the subtle structure of vortex could be identified relatively easily by numerical method, which could provide small enough contours.

When the phase angle jumps back to the negative part, the vortex wake exhibits a $2 P$ pattern, as shown in Figure 9. The two vortices in a vortex pair have almost equal strength. However, this $2 P$ mode is different from that in Figure 5 in that the vortices in a pair are separated from each other when two pairs of vortices shed alternatively behind the cylinder.

In this section, the new distinct mode pattern $2 P_{O}$ is verified by numerical simulations. Moreover, the amplitude $A^{*}$ and wavelength $\lambda^{*}$ where the $2 P$ and $2 P_{O}$ modes are observed in the simulations are located in the corresponding $2 P$ and $2 P_{O}$ regions of Morse and Williamson [27]. In the overlapping area, the vortex mode is not fixed, the vortex mode changes between the two modes $2 P$ and $2 P_{O}$, and even the cylinder is vibrating with a fixed frequency and amplitude.

As mentioned above in the introduction, for the typical model of low mass ratio, the amplitude responses are comprised by three branches: initial branch, upper branch, and lower branch. The vortex pattern is gradually turning from the $2 S$ mode in the initial branch into the $2 P$ mode in the upper and lower branches, while the $2 P_{\text {upper }}$ mode of upper branch is different from the $2 P_{\text {lower }}$ mode 


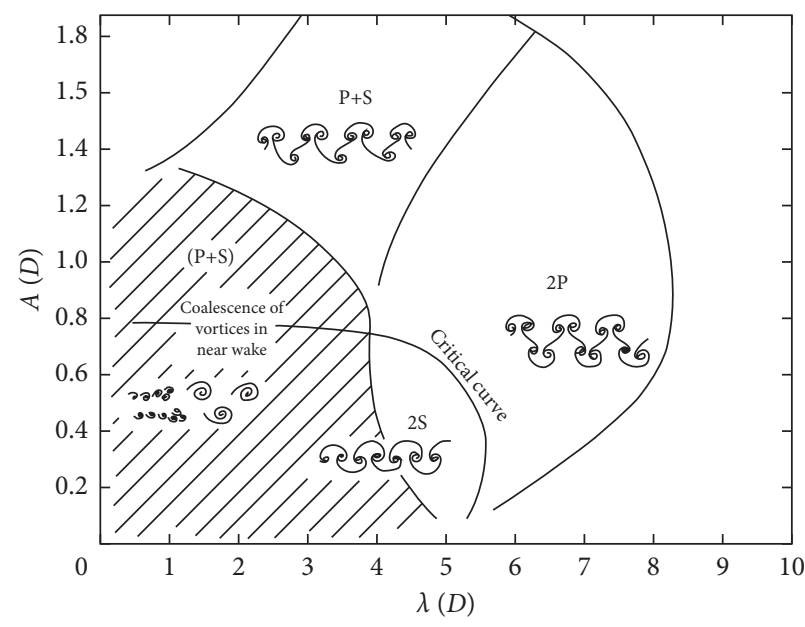

(a)

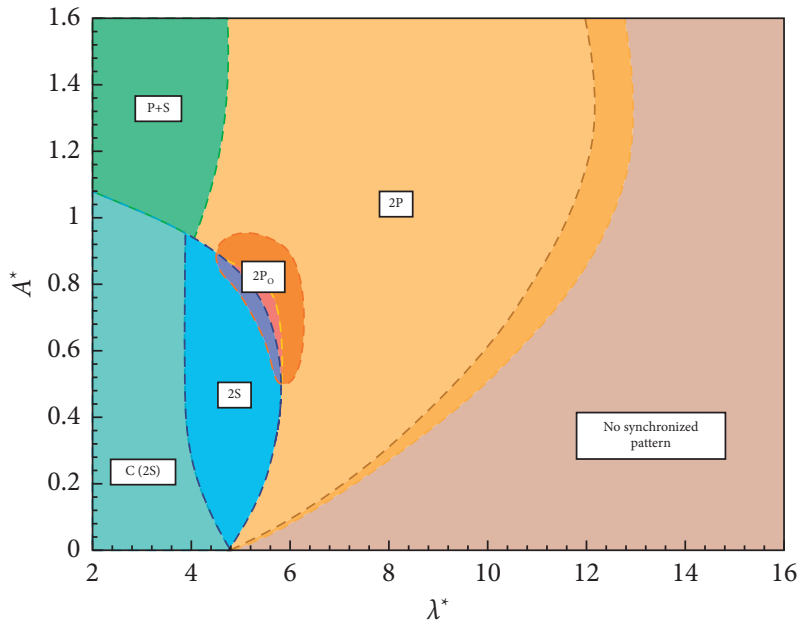

(b)

FIGURE 3: (a) Boundaries of wake modes identified by Williamson and Roshko [18]. (b) Boundaries of wake modes identified by Morse and Williamson [27].
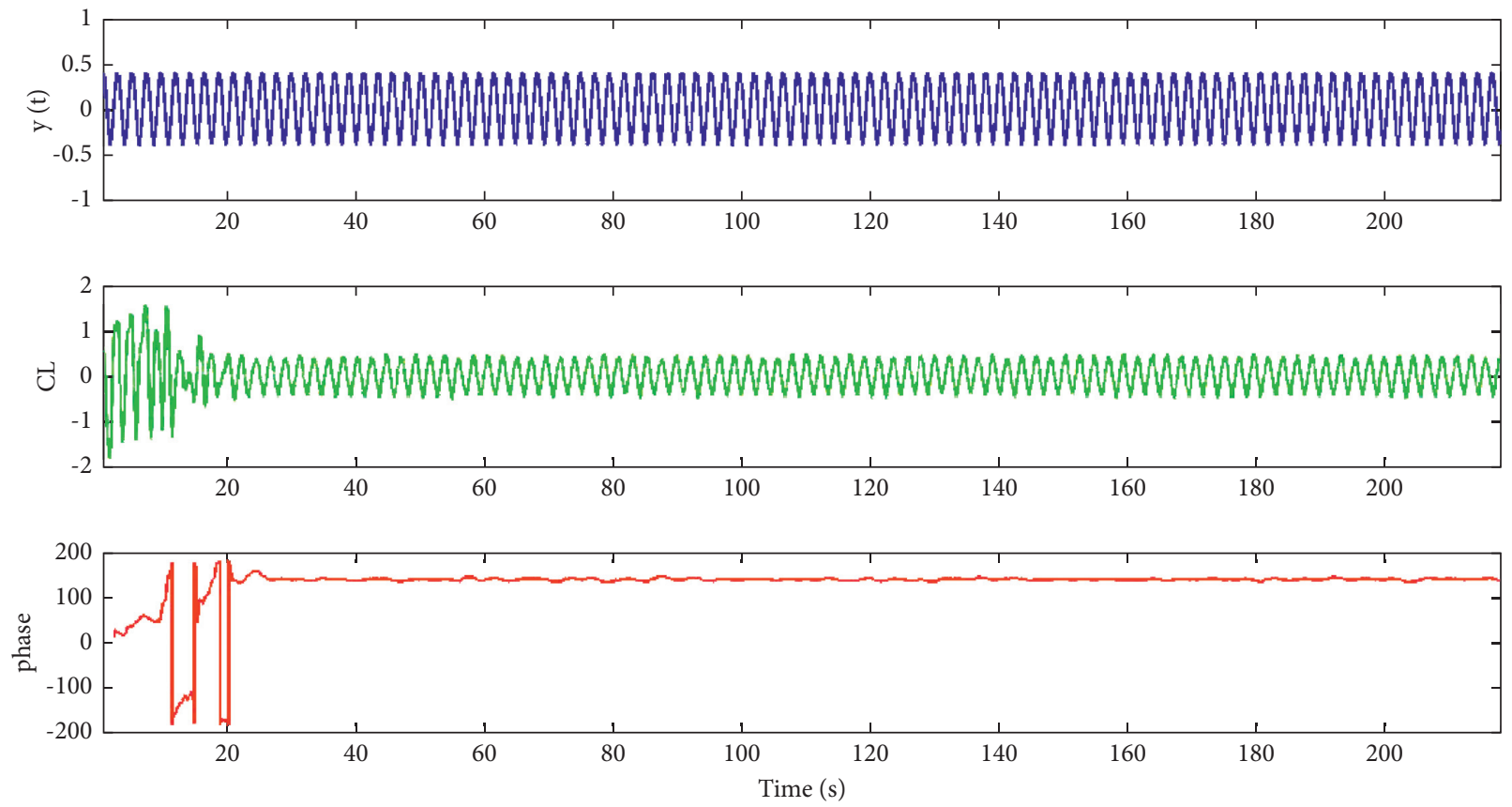

FIgURE 4: The cylinder motions, lift coefficient, and phase angle in time-domain at $A^{*}=0.4, \lambda^{*}=6.2$.

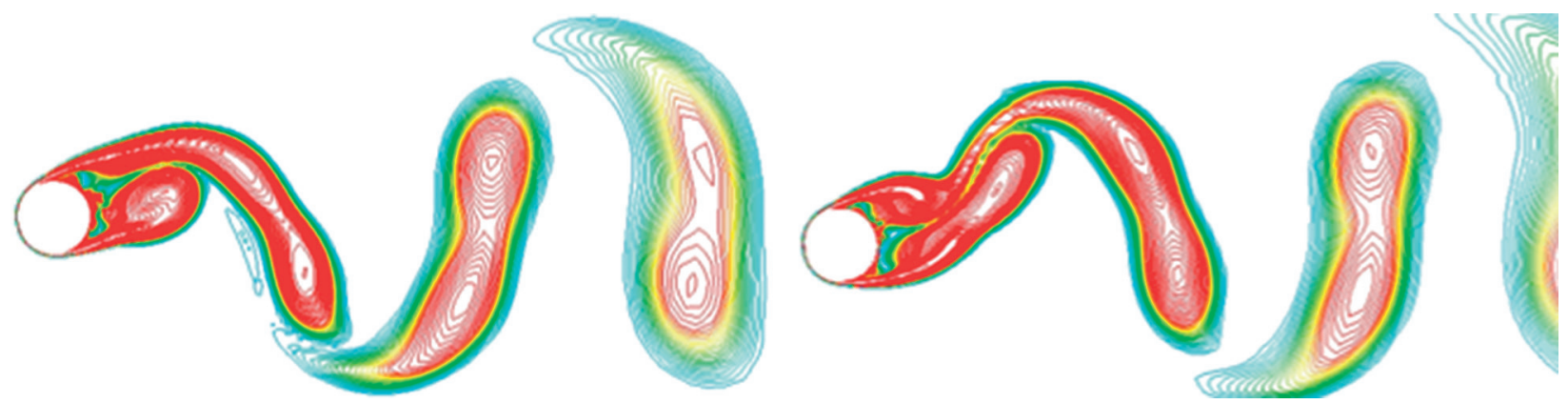

Figure 5: Vorticity contours of $2 P$ mode in one vibration period at $A^{*}=0.4, \lambda^{*}=6.2$. 

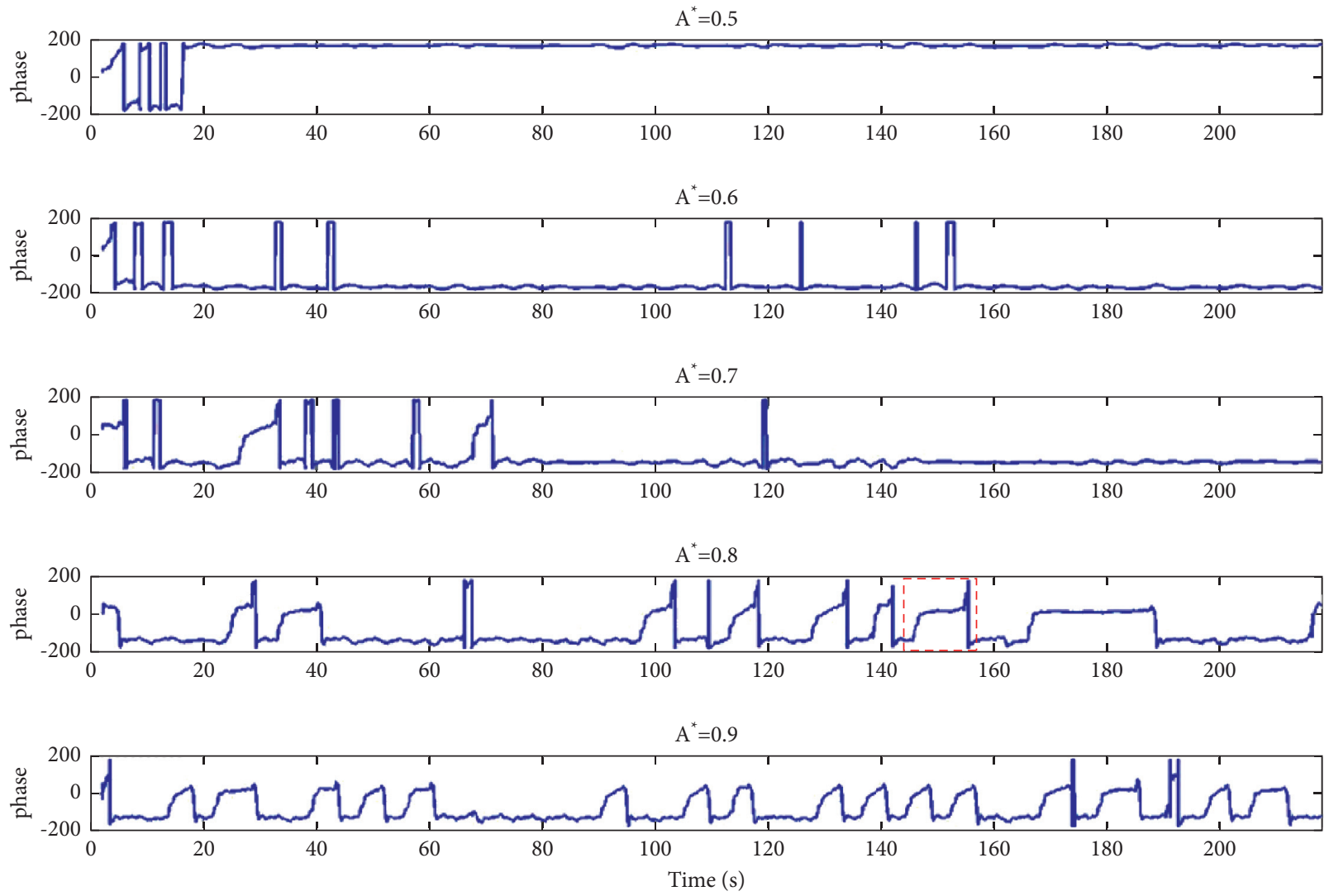

Figure 6: Vortex field of $2 P_{O}$ mode at the positive part of phase angle.

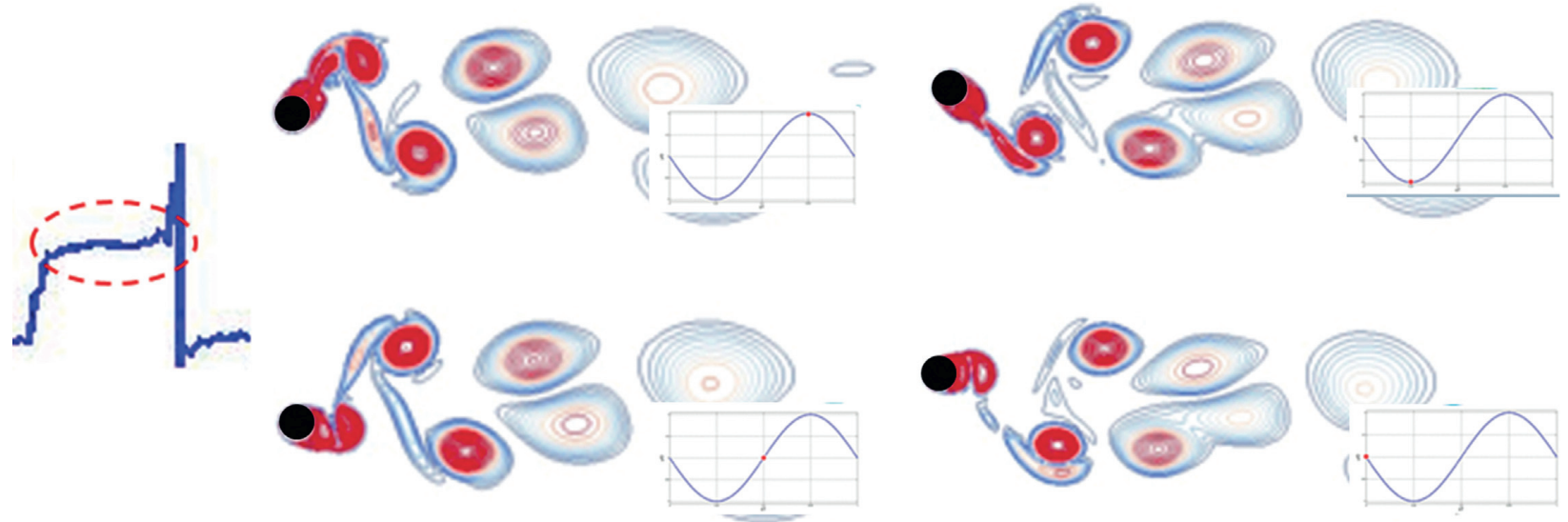

FIgURE 7: Phase angle at different amplitude ratios varying from 0.5 to 0.9 .

of lower branch, as shown in Figure 10. The newly found $2 P_{O}$ vortex-formation mode is equivalent to the " $2 P_{\text {upper }}$ " mode found by Govardhan and Williamson [28]. Khalak \& Williamson [12] found that the intermittent switches between upper branch and lower branch are observed in the low-mass ratio studies, as shown in Figure 11. The overlap regions can be used to explain the intermittent switch, because, in the overlap regions, the vortex mode switches intermittently between two distinct modes $2 P$ and $2 P_{O}$, which is closely related to the intermittent switch existing between the upper and lower branches. When the phase angle $\varphi$ is positive, the vortex mode appears $2 P_{O}$, the energy transfers from fluid to cylinder, and it will yield positive excitation. And the amplitude response is located in the upper branch. By contrast, the negative phase angle $\varphi$ means the negative excitation from cylinder to fluid. The amplitude response is located in the lower branch. 


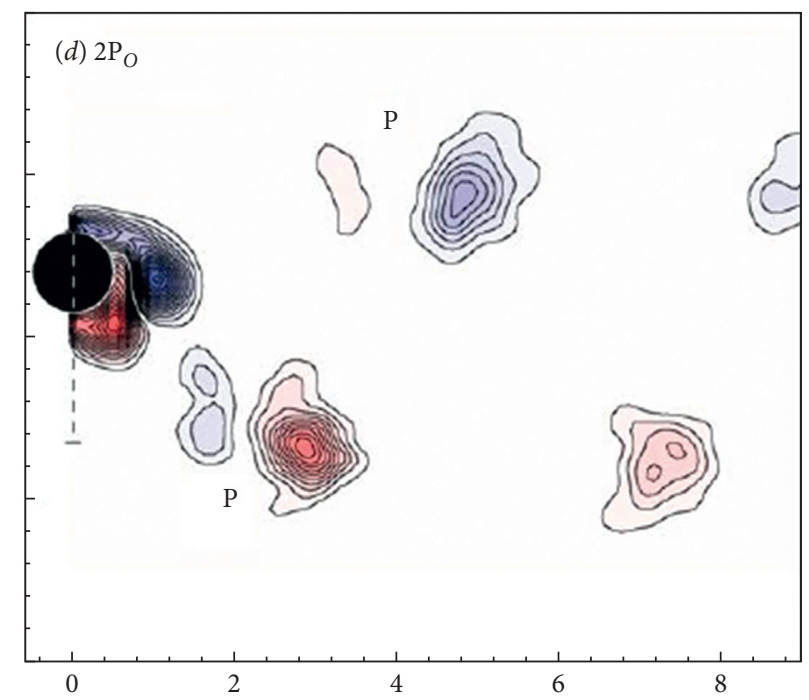

Figure 8: $2 P_{O}$ mode vortex field by using DPIV, Morse and Williamson [27].
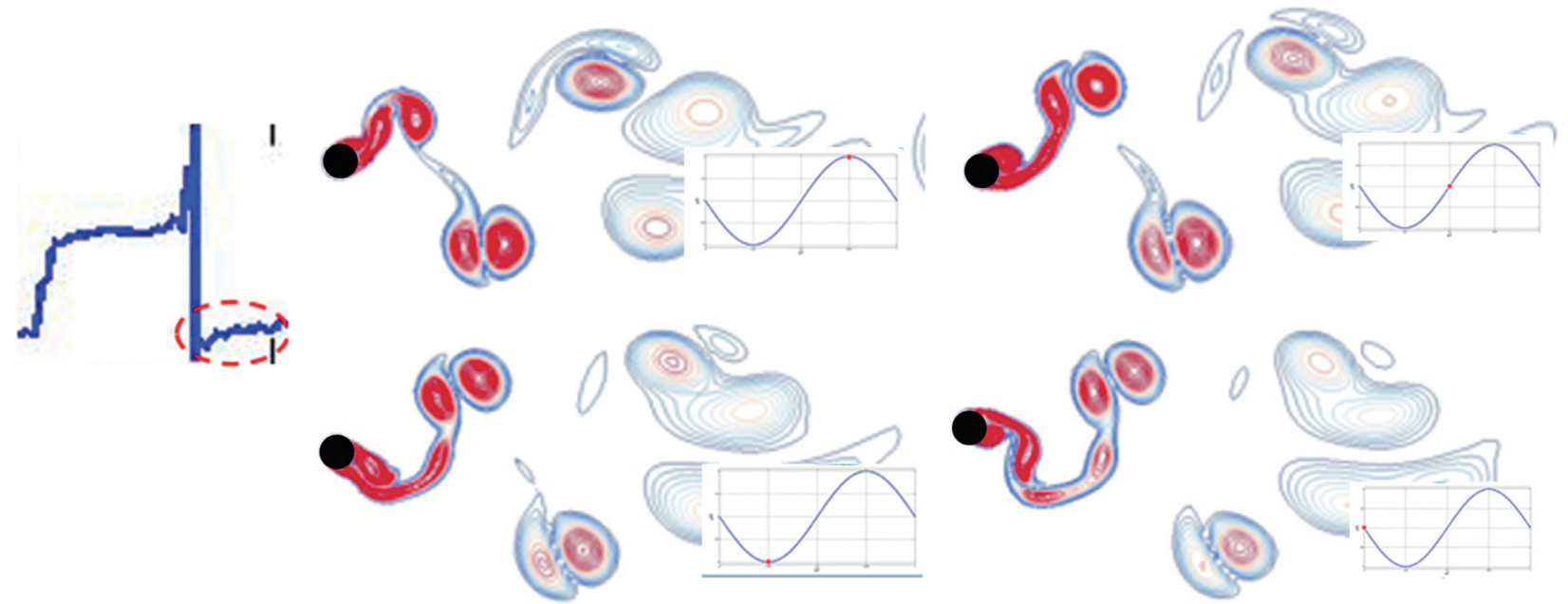

Figure 9: Vortex field of $2 P$ mode at the negative part of phase angle.

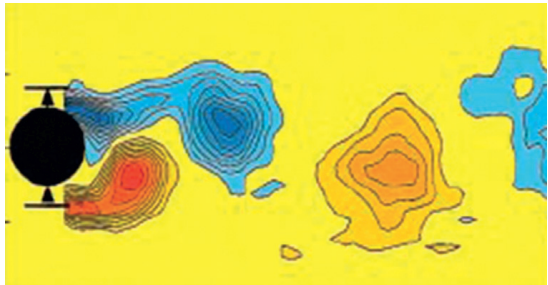

(a)

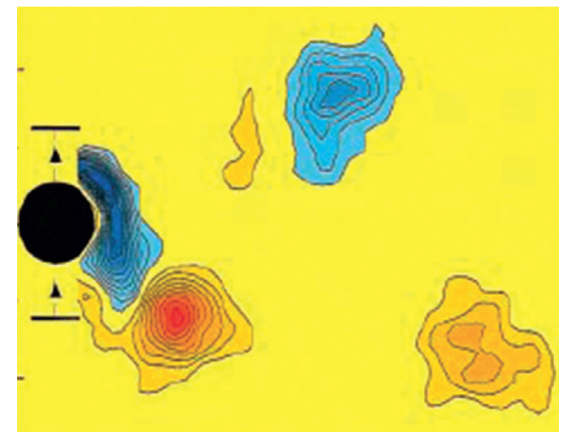

(b)

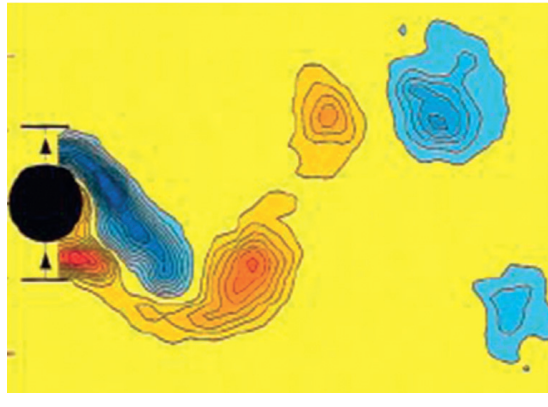

(c)

FIGURE 10: Vortex patterns of the three VIV response branches, from Govardhan and Williamson [28]. (a) Initial branch. (b)Upper branch. (c)Lower branch. 


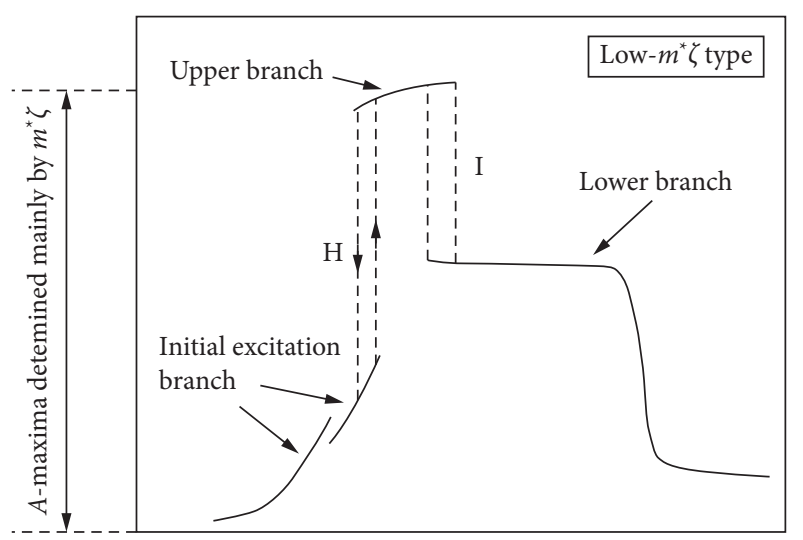

FIGURE 11: Intermittent switch (I) between upper branch and lower branch.

3.3. Prediction Results and Discussion. In this section, we try to predict free-vibration response of Govardhan and Williamson [28], as shown in Figure 12, by the above prediction method. In order to predict the response by controlledvibration data, the inlet velocity $U$ remains the same, and forced oscillating frequency $f_{\text {ex }}$ is changed. Thus, we can get different amplitude responses at different $\lambda^{*}=U / f_{\text {ex }} D$.

The availability of the force coefficient in phase with velocity $C_{L 0} \sin \varphi$ enables us to predict the response of a freevibration cylinder. We can simply solve numerically for amplitude $\left(A^{*}\right)$ and frequency $\left(f^{*}\right)$ and build up a response plot at a given $m^{*} \zeta$. Continuing $\$ 3.2$ wake modes and transitions study above, we try to predict the amplitude response at $\lambda^{*}=6.2$. Table 4 gives the amplitude of the lift coefficient $C_{L 0}$, the phase angle $\varphi$, and the force coefficient in phase with velocity $C_{L 0} \sin \varphi$ at different amplitude ratio $A^{*}$ and constant wavelength $\lambda^{*}=6.2$ of the forced oscillation. It is particularly worth mentioning that the phase angle listed in Table 4 is the stable angle. In the overlap region, there exist two phase angles. The average negative angle is the stable angle, and the jumping positive angle is the unstable angle.

After we have got the value of the force coefficient in phase with the velocity, we can readily use our controlledvibration data to predict the response of a freely vibrating cylinder at different mass damping $m^{*} \zeta=0.0,0.059,0.187,0.252$, in the example of Figure 13. The red circles in Figure 13 are the values of the term $\left(C_{L 0} \sin \varphi\right)$ at different forced amplitude ratio $A^{*}$. The blue asterisks denote the intersections of two lines corresponding to the right and left terms in prediction equation (16). We find close agreement with the measured free vibration amplitude response (taken from [28], from the error analysis shown in Table 5).

For the low-mass-damping $m^{*} \zeta$ case, there are threebranch responses, named initial branch, upper branch, and lower branch. In the free vibration of Figure 13, there exists an intermittent switch between the upper and lower branches at $\lambda^{*}=6.2$. The point $\lambda^{*}=6.2$ is located in the initial part of lower branch. According to the introduction of the part $\$ 3.2$, the phase angle $\varphi$ is unstable at $\lambda^{*}=6.2$, which is accompanied by the abrupt jump from negative angle to positive angle. When the phase angle $\varphi$ is negative, the

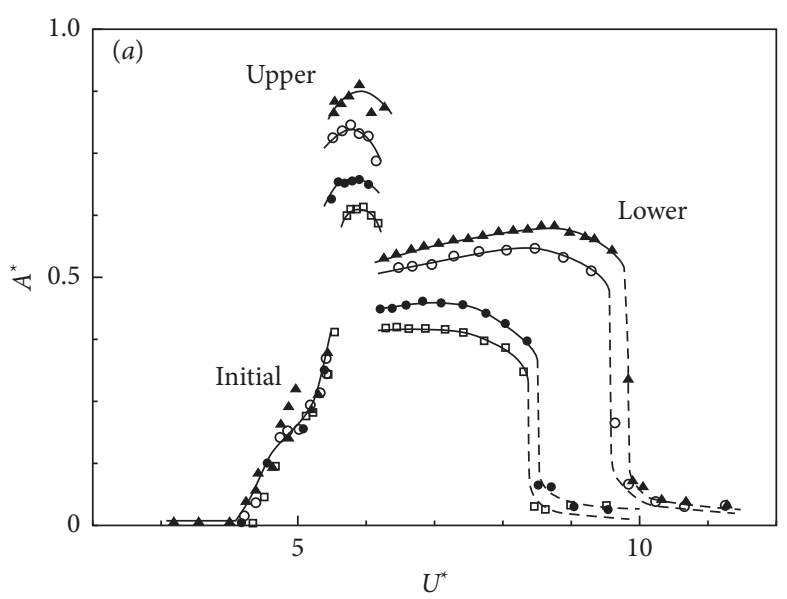

Figure 12: Amplitude response plots at a constant Reynolds number $(\operatorname{Re} \approx 4000)$ at different mass damping values $\left(m^{*}+C_{A}\right) \zeta=\alpha=0.000 ; 0.059 ; 0.187 ; 0.252$, taken from Govardhan and Williamson [28]. $\boldsymbol{\Delta}, \alpha=0.000 ; O, \alpha=0.059 ; \boldsymbol{\bullet}, \alpha=0.187$; $\square, \alpha=0.252$.

TABLE 4: Forced oscillating response at different amplitude ratio $A^{*}$ at $\lambda^{*}=6.2$.

\begin{tabular}{ccccc}
\hline$A^{*}$ & $\lambda^{*}$ & $C_{L 0}$ & $\varphi$ & $C_{L 0} \sin \varphi$ \\
\hline 0.4 & 6.2 & 0.4478 & 107.6156 & 0.4268 \\
0.5 & 6.2 & 0.5083 & 159.6684 & 0.1766 \\
0.6 & 6.2 & 0.6030 & -159.6776 & -0.2094 \\
0.7 & 6.2 & 0.8782 & -126.7985 & -0.7032 \\
0.8 & 6.2 & 1.2734 & -112.6470 & -1.1752 \\
0.9 & 6.2 & 1.6000 & -110.9883 & -1.4939 \\
\hline
\end{tabular}

response amplitude $A^{*}$ is located in lower branch. When the phase angle $\varphi$ jumps to the positive, extra energy is transported from fluid to cylinder. Then, it will cause a larger amplitude response, which is located in the upper branch. But the state is very unstable, and the response will come down to the lower branch again.

Analysis of wake modes and transitions in part $\$ 3.2$ shows that if the vortex mode is $2 P$, then there will be one stable equilibrium at the lower branch amplitude. However, this equilibrium will only persist if the vortex-formation mode continues to be $2 P$. If the wake switches to the $2 P_{O}$ mode, the fluid excitation will raise. This will cause the energy into the system to be more than the energy dissipated by damping, and therefore, the amplitude will increase until a new stable equilibrium for the $2 P_{O}$ mode is reached, corresponding to an upper branch amplitude. Later in time, the vortex-formation mode could possibly switch back to $2 P$, causing the amplitude to decrease back toward the lower branch amplitude. In this way, the amplitude could switch intermittently between the upper and lower branches.

For VIV problems, the maximum displacement of cylinder is our focus. From Figure 12, we find that the maximum displacement is around $U^{*}=6.0$. So, we carry out several numerical simulations of controlled oscillating cylinder undergoing a constant vibration frequency $\lambda^{*}=6.0$, 


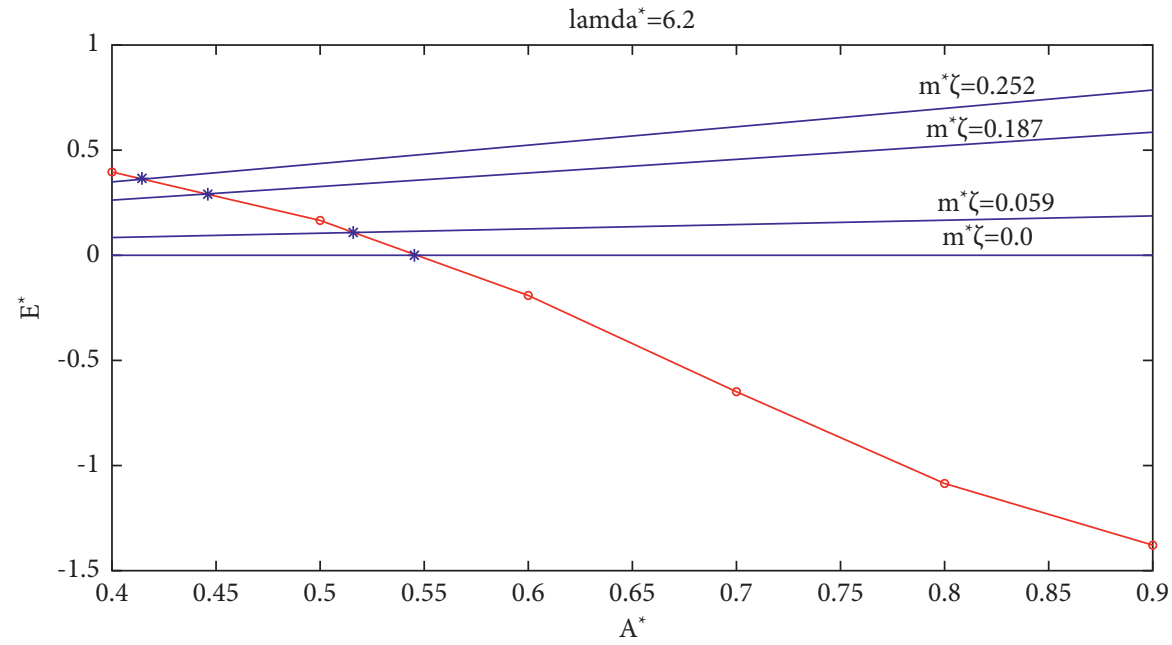

Figure 13: Prediction of amplitude response $A^{*}$ at different mass damping when $\lambda^{*}=6.2$.

TABle 5: Comparison of $A^{*}$ at $\lambda^{*}=6.2$ with experiment results.

\begin{tabular}{lccc}
\hline$m^{*} \zeta$ & $A^{*} \_$predict & $A^{*} \_$experiment of Govardhan and Williamson [28] & Error (\%) \\
\hline 0.0 & 0.4150 & 0.3958 & 4.9 \\
0.059 & 0.4464 & 0.4380 & 1.9 \\
0.187 & 0.5164 & 0.5092 & 1.4 \\
0.252 & 0.5457 & 0.5356 & 1.9 \\
\hline
\end{tabular}

TABLE 6: Forced oscillating response at different amplitude ratio $A^{*}$ at $\lambda^{*}=6.0$.

\begin{tabular}{llccc}
\hline$A^{*}$ & $\lambda^{*}$ & $C_{L 0}$ & $\varphi$ & $C_{L 0} \sin \varphi$ \\
\hline 0.4 & 6.0 & 0.4480 & 107.5006 & 0.4273 \\
0.5 & 6.0 & 0.5798 & 111.2018 & 0.5184 \\
0.6 & 6.0 & 0.6748 & 140.4302 & 0.4298 \\
0.7 & 6.0 & 0.8774 & 168.8271 & 0.1700 \\
0.8 & 6.0 & 1.2751 & -164.1542 & -0.3482 \\
0.9 & 6.0 & 1.6286 & -156.4862 & -0.6498 \\
\hline
\end{tabular}

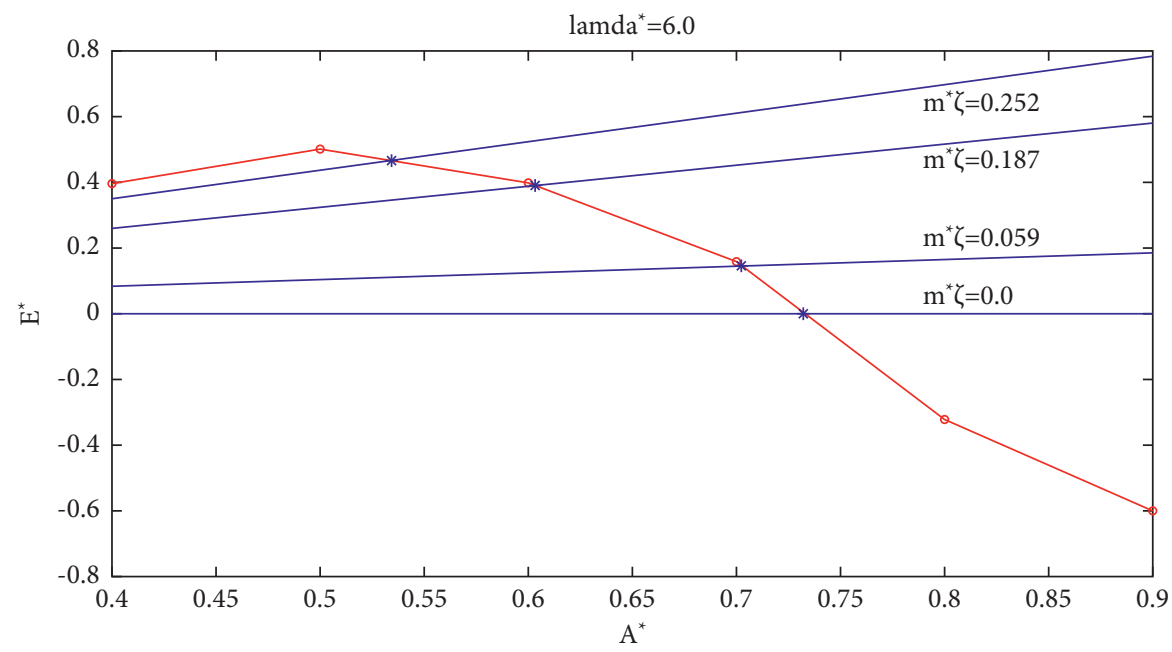

Figure 14: Prediction of amplitude response $A^{*}$ at different mass damping when $\lambda^{*}=6.0$. 
TABLE 7: Comparison of $A^{*}$ at $\lambda^{*}=6.0$ with experiment results.

\begin{tabular}{lccc}
\hline$m^{*} \zeta$ & $A^{*}$ _predict & $A^{*} \_$experiment of Govardhan and Williamson [28] & Error (\%) \\
\hline 0.0 & 0.7328 & 0.8707 & 15.8 \\
0.059 & 0.7030 & 0.7836 & 10.3 \\
0.187 & 0.6037 & 0.6912 & 12.6 \\
0.252 & 0.5350 & 0.6332 & 15.5 \\
\hline
\end{tabular}

TABLE 8: Forced oscillating response at different amplitude ratio $A^{*}$ at $\lambda^{*}=5.0$.

\begin{tabular}{llccc}
\hline$A^{*}$ & $\lambda^{*}$ & $C_{L 0}$ & $\varphi$ & $C_{L 0} \sin \varphi$ \\
\hline 0.1 & 5.0 & 0.5767 & 101.5021 & 0.5651 \\
0.2 & 5.0 & 0.6198 & 112.1354 & 0.5741 \\
0.3 & 5.0 & 0.7451 & -176.3427 & -0.0475 \\
0.4 & 5.0 & 0.8267 & -161.7941 & -0.2583 \\
0.5 & 5.0 & 0.9762 & -153.2176 & -0.4399 \\
0.6 & 5.0 & 1.1301 & -145.5132 & -0.6399 \\
\hline
\end{tabular}

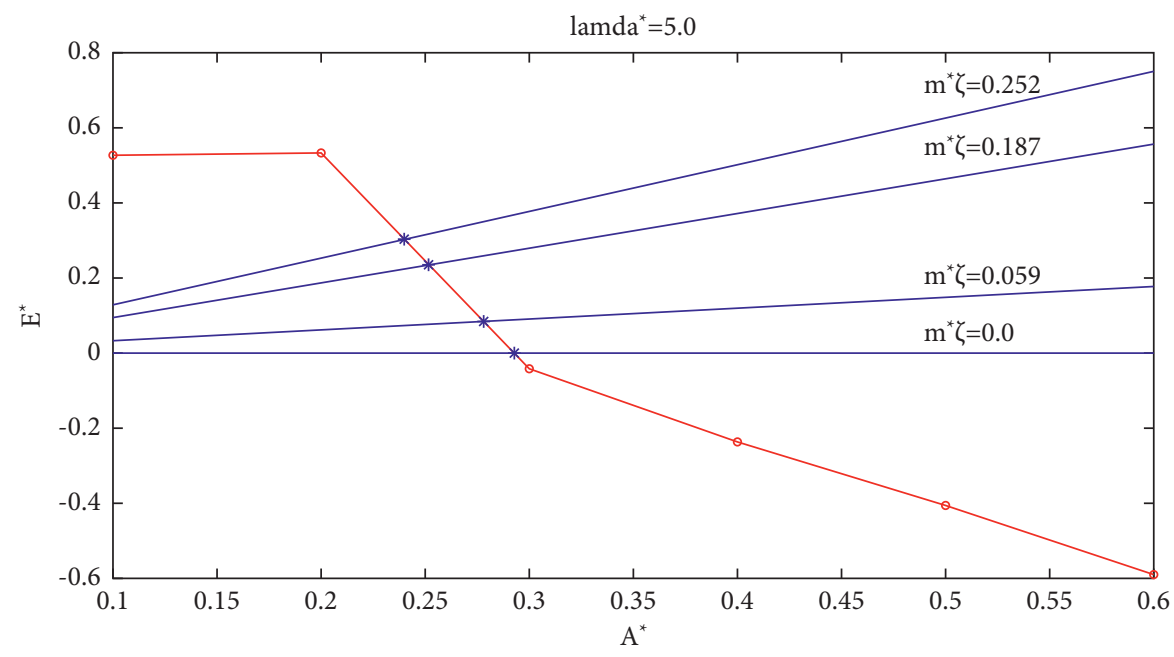

Figure 15: Prediction of amplitude response $A^{*}$ at different mass damping when $\lambda^{*}=5.0$.

TABle 9: Comparison of $A^{*}$ at $\lambda^{*}=5.0$ with experiment results.

\begin{tabular}{lccc}
\hline$m^{*} \zeta$ & $A^{*} \_$predict & $A^{*} \_$experiment of Govardhan and Williamson [28] & Error (\%) \\
\hline 0.0 & 0.292 & 0.28 & 4.2 \\
0.059 & 0.270 & 0.22 & 22.7 \\
0.187 & 0.251 & 0.22 & 14.1 \\
0.252 & 0.239 & 0.22 & 8.6 \\
\hline
\end{tabular}

and different amplitude $A^{*}$ varied from 0.4 to 0.9 in this paper. And the forced oscillating results are listed in Table 6.

The prediction of $A^{*}$ at $\lambda^{*}=6.0$ is shown in Figure 14 and Table 7 . Although the prediction results offer less accuracy than that at $\lambda^{*}=6.2$, we believe that the error would be reduced if the distribution of the forced vibration amplitude ratio $A^{*}$ is refined, e.g., with a resolution of 0.01 .

The above two cases have verified the amplitude responses in the upper and lower branches. In order to verify the good versatility of the prediction method, we try to predict the amplitude responses in the initial branches. We carry out several numerical simulations of controlled oscillating cylinder undergoing a constant vibration frequency $\lambda^{*}=6.0$, because the amplitude in the initial branch is small. Different amplitudes $A^{*}$ are chosen varying from 0.1 to 0.6 , and the forced oscillating results are listed in Table 8.

The prediction of $A^{*}$ at $\lambda^{*}=5.0$ is shown in Figure 15 . The prediction results and comparison with the 
experimental results at $\lambda^{*}=5.0$ are shown in Table 9. The experimental results are very close to each other. Especially for $m^{*} \zeta=0.059,0.187,0.252$, all the amplitude responses are the same $A^{*}=0.22$. This is because the experiment accuracy is limited. It is difficult to distinguish the amplitude difference when the amplitude response is small. However, the predicted amplitudes by numerical method are discrete distribution. The smaller the mass damping is, the larger the amplitude response is. The upper and lower bounds of the predicted amplitudes are close to the experimental results, but the intermediate values are quite different, which may be the error caused by the experiment accuracy.

\section{Conclusion}

In this paper, numerical simulations of a forced oscillating cylinder at a wide range of oscillating amplitudes and frequencies at $\operatorname{Re}=4000$ were performed by a newly developed solver viv-FOAM-SJTU. First, grid convergence study was conducted to further validate the computational results. Four grids with different nodes around the cylinder and grid's height of first layer were applied. The convergence study was performed for a forced oscillating cylinder, and good convergence was achieved. Complex demodulation was chosen to analyze the spatial-temporal phase relationship between the forces and cylinder displacement. The time history of the phase angle was presented to understand the mechanism of vortex mode transitions. Abrupt jump from a negative value to a positive value was observed for the phase angle. Accompanied by the jumping of the phase angle, the vortex wake pattern also changed. By numerical simulations, we identified a new distinct mode of vortex formation. This new vortex model was comprised of two pairs of vortices formed per cycle, where the secondary vortex in each pair was much weaker than the primary vortex. This vortex mode was defined as $2 P_{O}$, which was closely related to the intermittent switch existing between the upper and lower branches. The good details of vortex after cylinder and the vortex propagating downstream were presented. The simulation region calculated in this part was in well agreement with the "OVERLAP" region of Morse and Williamson [27] experiments. Prediction function of a free-vibration cylinder based on vibration equation of one-degree-of-freedom was derived. Controlled-vibration data was used to predict the response of a freely vibrating cylinder at different mass damping $m^{*} \zeta$. Take the free vibration of Govardhan and Williamson [28] as an example, we had successfully predicted the VIV response by forced vibration data at three typical cases $\lambda^{*}=5.0,6.0,6.2$ located in the initial, upper, and lower branches separately. The method predicted the amplitude response with acceptable accuracy. Future work included building up an entire response plot for all reduced velocity and investigating whether the prediction approach is held for all Reynolds number.

\section{Data Availability}

The data used to support the findings of this study are included within the article.

\section{Conflicts of Interest}

The authors declare that they have no conflicts of interest.

\section{Acknowledgments}

This work was supported by the Science and Technology Program of Guangzhou (202002030424), Research Program for Higher Education of Guangzhou Education Bureau (202032786), Innovation and Strong University Project of Guangdong Education Bureau (2019KTSCX123 and 2020KTSCX107), and Technological Innovation Fund for of Guangdong Province--Climb Plan (pdjh2020b0447), to which the authors are most grateful.

\section{References}

[1] C. H. K. Williamson, "Vortex dynamics in the cylinder wake," Annual Review of Fluid Mechanics, vol. 28, pp. 477-539, 1996.

[2] C. H. K. Williamson and R. Govardan, "Vortex-induced vibration," Annual Review of Fluid Mechanics, vol. 36, pp. 413-455, 2004.

[3] C. H. K. Williamson and R. Govardan, "A brief review of recent results in vortex-induced vibrations," Journal of Wind Engineering, vol. 96, pp. 713-735, 2008.

[4] T. Sarpkaya, "A critical review of the intrinsic nature of vortex-induced Vibrations," Journal Fluids and Strucures.vol. 19, pp. 389-447, 2004.

[5] R. D. Gabbai and H. Benaroya, "An overview of modeling and experiments of vortex-induced vibration of circular cylinders," Journal of Sound and Vibration, vol. 282, no. 3-5, pp. 575-616, 2005.

[6] P. W. Bearman, "Circular cylinder wakes and vortex-induced vibrations," Journal of Fluids and Structures, vol. 27, no. 5-6, pp. 648-658, 2011.

[7] X. D. Wu, F. Ge, and Y. S. Hong, "A review of recent studies on vortex-induced vibrations of long slender cylinders," Journal of Fluids and Structures, vol. 28, pp. 292-308, 2012.

[8] C. C. Feng, "The measurements of vortex-induced effects in flow past a stationary and oscillating circular and d-section cylinders," Masters thesis, University of British, Vancouver, Canada, 1968.

[9] A. Khalak and C. H. K. Williamson, "Dynamics of a hydroelastic cylinder with very low mass and damping," Journal of Fluids and Structures, vol. 10, no. 5, pp. 455-472, 1996.

[10] A. Khalak and C. H. K. Williamson, "Fluid forces and dynamics of a hydroelastic structure with very low mass and damping," Journal of Fluids and Structures, vol. 11, no. 8, pp. 973-982, 1997a.

[11] A. Khalak and C. H. K. Williamson, "Investigation of relative effects of mass and damping in vortex-induced vibration of a circular cylinder," Journal of Wind Engineering and Industrial Aerodynamics, vol. 69-71, pp. 341-350, 1997b.

[12] A. Khalak and C. H. K. Williamson, "Motions, forces and mode transitions in vortex-induced vibrations at low massdamping," Journal of Fluids and Structures, vol. 13, no. 7-8, pp. 813-851, 1999.

[13] N. Jauvtis and C. H. K. Williamson, "The effect of two degrees of freedom on vortex-induced vibration at low mass and damping," Journal of Fluid Mechanics, vol. 509, pp. 23-62, 2004. 
[14] Navrose and M. Sanjay, "Free vibrations of a cylinder: 3-D computations at $\mathrm{Re}=1000$," Journal of Fluids and Structures, vol. 41, pp. 109-118, 2013.

[15] H. Al-Jamal and C. Dalton, "Vortex induced vibrations using large Eddy simulation at a moderate Reynolds number," Journal of Fluids and Structures, vol. 19, no. 1, pp. 73-92, 2004.

[16] D. Lucor, J. Foo, and G. E. Karniadakis, "Vortex mode selection of a rigid cylinder subject to VIV at low massdamping," Journal of Fluids and Structures, vol. 20, no. 4, pp. 483-503, 2005.

[17] J. B. V. Wanderley, G. H. B. Souza, S. H. Sphaier, and C. Levi, "Vortex-induced vibration of an elastically mounted circular cylinder using an upwind TVD two-dimensional numerical scheme," Ocean Engineering, vol. 35, no. 14-15, pp. 1533-1544, 2008.

[18] C. H. K. Williamson and A. Roshko, "Vortex formation in the wake of an oscillating cylinder," Journal of Fluids and Structures, vol. 2, pp. 355-381, 1988.

[19] T. Nishihara, S. Kaneko, and T. Watanabe, "Characteristics of fluid dynamic forces acting on a circular cylinder oscillated in the streamwise direction and its wake patterns," Journal of Fluids and Structures, vol. 20, pp. 505-518, 2005.

[20] D. Jeon and M. Gharib, "On circular cylinders undergoing two-degree-of-freedom forced motions," Journal of Fluids and Structures, vol. 15, no. 3-4, pp. 533-541, 2001.

[21] S. Atluri, V. K. Rao, and C. Dalton, "A numerical investigation of the near-wake structure in the variable frequency forced oscillation of a circular cylinder," Journal of Fluids and Structures, vol. 25, no. 2, pp. 229-244, 2009.

[22] S. Dong and G. E. Karniadakis, "DNS of flow past a stationary and oscillating cylinder at," Journal of Fluids and Structures, vol. 20, no. 4, pp. 519-531, 2005.

[23] W. W. Zhao and D. C. Wan, "Detached-eddy simulation of flow past tandem cylinders," Applied Mathematics and Mechanics, vol. 37, no. 12, pp. 1272-1281, 2016.

[24] W. W. Zhao and D. C. Wan, "Numerical study of 3D flow past a circular cylinder at subcritical Reynolds number using SSTDES and SST-URANS," Chinese Journal of Hydrodynamics, vol. 31, no. 1, pp. 1-8, 2016.

[25] B. Fu, L. Zou, and D. Wan, "Numerical study of vortex-induced vibrations of a flexible cylinder in an oscillatory flow," Journal of Fluids and Structures, vol. 77, pp. 170-181, 2018.

[26] D. Deng, WW. Zhao, and D. C. Wan, "Vortex-induced vibration prediction of a flexible cylinder by three-dimensional strip model," Ocean Engineering, vol. 205, Article ID 107318, 2020.

[27] T. L. Morse and C. H. K. Williamson, "Prediction of vortexinduced vibration response by employing controlled motion," Journal of Fluid Mechanics, vol. 634, pp. 5-39, 2009.

[28] R. N. Govardhan and C. H. K. Williamson, "Defining the 'modified Griffin plot' in vortex-induced vibration: revealing the effect of Reynolds number using controlled damping," Journal of Fluid Mechanics, vol. 561, pp. 147-180, 2006.

[29] H. Jasck, Error analysis and estimation for the finite volume method with applications to fluid flows, Ph.D. thesis, Imperial College, London, UK, 1996.

[30] H. Rusche, Computational Fluid Dynamics of Dispersed Twophase Flows at High Phase Fractions, Ph.D. thesis, Imperial College, London, UK, 2002. 